\title{
Editorial: Recent Developments in Therapies and Diagnostic Tools for Melanoma and Non-melanoma Skin Cancer
}

\author{
Taku Fujimura ${ }^{1}$, Yasuhiro Fujisawa ${ }^{2}$, Atsushi Otsuka ${ }^{3}$ and Nikolas K. Haass ${ }^{4 *}$ \\ ${ }^{1}$ Department of Dermatology, Tohoku University Graduate School of Medicine, Sendai, Japan, ${ }^{2}$ Department of Dermatology, \\ University of Tsukuba, Tsukuba, Japan, ${ }^{3}$ Department of Dermatology, Kyoto University Graduate School of Medicine, Kyoto, \\ Japan, ${ }^{4}$ The University of Queensland Diamantina Institute, The University of Queensland, Brisbane, QLD, Australia
}

Keywords: biomarker (BM), immune checkpoint antibodies, dermoscopy (DS), skin imaging, T-cell lymphoma cutaneous, sentinel lymph node biopsy (SLNB), deep-learning (DL), convolutional neural network (CNN)

Editorial on the Research Topic

Recent Developments in Therapies and Diagnostic Tools for Melanoma and Non-melanoma Skin Cancer

\section{OPEN ACCESS}

Edited and reviewed by: Robert Gniadecki,

University of Alberta, Canada

*Correspondence:

Nikolas K. Haass

n.haass1@uq.edu.au

Specialty section:

This article was submitted to

Dermatology,

a section of the journal

Frontiers in Medicine

Received: 01 October 2020

Accepted: 13 October 2020

Published: 12 November 2020

Citation:

Fujimura T, Fujisawa Y, Otsuka A and

Haass NK (2020) Editorial: Recent

Developments in Therapies and

Diagnostic Tools for Melanoma and

Non-melanoma Skin Cancer.

Front. Med. 7:613152.

doi: 10.3389/fmed.2020.613152

\section{UPDATES ON BIOMARKERS FOR IMMUNE CHECKPOINT THERAPY OF ADVANCED MELANOMA}

In the past decade, there has been a paradigm shift for the treatment of skin cancer, especially of advanced melanoma, due to the unprecedented success of MAPK pathway and immune checkpoint inhibitors (MAPKi, ICi). In this Research Topic, we focus on the latter. The most commonly used ICi are monoclonal antibodies targeting cytotoxic T-lymphocyte-associated protein 4 (CTLA-4; ipilimumab), programmed cell death 1 (PD-1; nivolumab and pembrolizumab) and, more recently, programmed death-ligand 1 (PD-L1; atezolizumab, durvalumab, and avelumab). In addition to multiple described resistance mechanisms to ICi (1), the lack of reliable biomarkers for both drug efficacy and immune-related adverse events (irAEs) has been a major clinical concern and is reviewed here extensively [Kambayashi et al.; Nakamura (a)]. Importantly, although many predictive biomarkers for both ICi tumor response and irAEs have been identified, no single biomarker has shown to be predictive on its own; therefore, a combination of multiple biomarkers should be utilized [Kambayashi et al.; Nakamura (a)]. Furthermore, irAEs and tumor response can be interconnected, but often are not; thus, irAEs or biomarkers for irAEs cannot be reliably used as biomarker for tumor response [Kambayashi et al.; Nakamura (a)]. Further studies are needed to improve biomarkers for predicting the efficacy of ICi treatment [Kambayashi et al.; Nakamura (a)]. To this end, Kümpers et al., show that PD-L1 expression on primary tumors and melanoma metastases is not associated with the clinical response of anti-PD1 antibodies, while several previous studies suggest an association of PD-L1 status and response to anti-PD1 antibodies (2). Instead, immune cell infiltration in the primary melanoma, measured by the Immunoscore, was associated with a significantly improved response to ICi in terms of increased overall survival 
(Kümpers et al.). Another research paper in this Research Topic suggests that baseline serum levels of CXCL5, which have been reported previously as a biomarker for autoimmune disease, could be a predictive marker for the efficacy of anti-PD1 antibodies (Fujimura, Sato et al.). While ICi were initially only used for definitive therapy, the field has quickly moved to adjuvant and, more recently, to neoadjuvant therapy (3). Combination of nivolumab plus ipilimumab $(\mathrm{N}+\mathrm{I})$ is amongst the most effective therapies against both BRAF-mutated and BRAF-wildtype advanced melanoma, but leads to a high frequency of irAEs [Nakamura (a)]. Fujimura, Kambayashi et al. show in a case report that $\mathrm{N}+\mathrm{I}$ combination therapy for BRAF-mutated advanced melanoma before primary tumor resection strikingly increased $\mathrm{CD} 8+$ cytotoxic $\mathrm{T}$ cells in the primary tumor, leading to induced anti-melanoma immune response in metastases in six different organs, but also induced serious AEs after administration of $\mathrm{N}+\mathrm{I}$ combination therapy (4). In addition, previous reports suggest that the efficacy of ipilimumab among patients with anti-PD1 antibody-resistant melanoma is extremely low after objective tumor progression (5). In summary, the optimization of immunotherapy using ICi is still challenging, but rapidly developing, and it is exciting to see how the landscape of melanoma biomarkers has changed within a decade (6).

\section{UPDATES ON SYSTEMIC THERAPIES FOR NON-MELANOMA SKIN CANCERS}

On the heels of the success of systemic therapies for melanoma, similar approaches are being tested in non-melanoma skin cancers (NMSC). Tanese et al. review systemic therapy options, including hedgehog inhibitors for basal cell carcinoma (BCC), EGFR inhibitors and ICi for cutaneous squamous cell carcinoma (cSCC), HER2 antagonists for extramammary Paget's disease (EMPD), ICi for Merkel cell carcinoma (MCC), and experimental approaches for skin adnexal carcinomas. They conclude that, emerging molecular targeting therapies are not necessarily effective for all NMSC patients. Development of further treatment options for NMSC is required, especially for rare forms of NMSC, such as skin adnexal carcinomas (Tanese et al.). A retrospective case series by Hiura et al. of 13 patients with unresectable cSCC demonstrates the potential advantage of continued chemotherapy after concurrent chemoradiotherapy (CCRT), which will be validated in a future study. Hidaka et al. discuss in a thought-provoking review the role that Aryl Hydrocarbon Receptor (AHR) plays in carcinogenesis and maintenance of skin cancers. AHR is a key modulator of UVR- and carcinogenic chemical-induced skin carcinogenesis and is also associated with the efficacy of MAPKi and ICi in melanoma (Hidaka et al.). Thus, the authors propose that the AHR system may provide a putative target for prevention and therapy of skin cancer (Hidaka et al.). Oka and Miyagaki review novel and future therapies for advanced Mycosis fungoides and Sézary syndrome covering a wide range of different drug classes. Most approaches showed limited efficacy. Thus, the authors recommend personalized therapy and call for creatively designed international clinical trials (Oka and Miyagaki).

\section{UPDATES ON DIAGNOSTIC TOOLS FOR MELANOMA AND NON-MELANOMA SKIN CANCER}

Dermoscopy has become an indispensable diagnostic tool for pigmented and unpigmented cutaneous lesions (7). Kato et al. review the role of dermoscopy in the diagnosis of melanoma and non-melanoma skin cancers including BCC, sebaceous carcinoma, actinic keratosis, Bowen's disease, cSCC, MCC, EMPD, and angiosarcoma. Oh et al. take skin imaging further by discussing the utility of ultrasound imaging, optical coherence tomography, confocal microscopy, and two-photon microscopy as diagnostic tools. Especially a combination of tools is advised to allow for highest resolution and highest imaging depth, which are usually reciprocal (Oh et al.). The principle of these devices is to analyze signals reflected or scattered from the skin. Indeed, the fact that autofluorescent structures within the skin (e.g., elastic fibers) can be co-imaged with highly crystalline triple-helix structures (e.g., collagen) utilizing the second harmonic generation phenomenon, which then can be quantified (8). Oh et al. suggest that the development of fluorescent probes will further improve the utility of these tools for the diagnosis and treatment of skin lesions. Fujisawa et al. take this further and discuss the strengths and limitations of a deep-learning technology using a convolutional neural network (CNN) for skin tumor diagnosis. They conclude that AI classifiers have dramatically improved over the last years and still keep improving and thus may gain sufficient sensitivity and specificity to bear the screening burden for detecting malignant skin tumors (Fujisawa et al.). Importantly, they emphasize that the advent of AI-based skin cancer diagnostic should be considered a useful assistance, rather than a threat to dermatologists (Fujisawa et al.).

Nakamura (b) discusses the new role of sentinel lymph node biopsy (SLNB) for invasive melanoma post DeCOG-SLT and MSLT-II, in the context of modern adjuvant and neoadjuvant therapy approaches.

Fujii and Kanekura review the methods for diagnosis of early stage T-Cell Lymphoma. They demonstrate that nextgeneration sequencing not only detects TCR clonality with superior sensitivity over conventional methods and is therefore better to diagnose early Mycosis fungoides, but also allows for temporal tracking of specific TCR clones and therefore better for assessment of progression or recurrence (Fujii and Kanekura).

\section{AUTHOR CONTRIBUTIONS}

$\mathrm{TF}$ and $\mathrm{NH}$ wrote the manuscript. All authors provided intellectual input to the editorial.

\section{ACKNOWLEDGMENTS}

$\mathrm{NH}$ was a Cameron fellow of the Melanoma and Skin Cancer Research Institute, Australia, and is currently funded 
by the PA Research Foundation, Meehan Project Grant (021174 2017002565), Cancer Council Queensland (Project
Grant APP1165618), and the Australian Research Council (ARC Discovery Grant DP200100177).

\section{REFERENCES}

1. Wilmott JS, Rizos H, Scolyer RA, Long GV. The "tricky business" of identifying mechanisms of resistance to anti-PD-1. Clin Cancer Res. (2017) 23:2921-3. doi: 10.1158/1078-0432.CCR-17-0669

2. Jessurun CAC, Vos JAM, Limpens J, Luiten RM. Biomarkers for response of melanoma patients to immune checkpoint inhibitors: a systematic review. Front Oncol. (2017) 7:233. doi: 10.3389/fonc.2017.00233

3. Blank CU, Rozeman EA, Fanchi LF, Sikorska K, van de Wiel B, Kvistborg P, et al. Neoadjuvant versus adjuvant ipilimumab plus nivolumab in macroscopic stage III melanoma. Nat Med. (2018) 24:1655-61. doi: 10.1038/s41591-018-0198-0

4. Kambayashi Y, Fujimura T, Kuroda H, Otsuka A, Irie H, Aiba S. Severe demyelinating neuropathy in an advanced melanoma patient treated with nivolumab plus ipilimumab combined therapy. Case Rep Oncol. (2020) 13:4747. doi: $10.1159 / 000506976$

5. Fujisawa Y, Yoshino K, Otsuka A, Funakoshi T, Uchi H, Fujimura T, et al. Retrospective study of advanced melanoma patients treated with ipilimumab after nivolumab: analysis of 60 Japanese patients. J Dermatol Sci. (2018) 89:606. doi: 10.1016/j.jdermsci.2017.10.009

6. Haass NK, Smalley KS. Melanoma biomarkers: current status and utility in diagnosis, prognosis, and response to therapy.

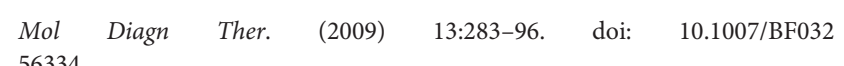

56334

7. Weber P, Tschandl P, Sinz C, Kittler H. Dermatoscopy of neoplastic skin lesions: recent advances, updates, and revisions. Curr Treat Options Oncol. (2018) 19:56. doi: 10.1007/s11864-018-0573-6

8. Tong PL, Qin J, Cooper CL, Lowe PM, Murrell DF, Kossard S, et al. A quantitative approach to histopathological dissection of elastin-related disorders using multiphoton microscopy. Br J Dermatol. (2013) 169:869-79. doi: 10.1111/bjd.12430

Conflict of Interest: The authors declare that the research was conducted in the absence of any commercial or financial relationships that could be construed as a potential conflict of interest.

Copyright $\odot 2020$ Fujimura, Fujisawa, Otsuka and Haass. This is an open-access article distributed under the terms of the Creative Commons Attribution License (CC $B Y)$. The use, distribution or reproduction in other forums is permitted, provided the original author(s) and the copyright owner(s) are credited and that the original publication in this journal is cited, in accordance with accepted academic practice. No use, distribution or reproduction is permitted which does not comply with these terms. 